\title{
Research on a Parallel Robot for Green Tea Flushes Plucking
}

\author{
Jun Chen ${ }^{1, a}$, Yong Chen ${ }^{2, b}{ }^{*}$, Xiaojun $\mathrm{Jin}^{3, \mathrm{c}}$, \\ Jun Che ${ }^{4, d}$, Feng $\mathrm{Gao}^{5, \mathrm{e}}$ and $\mathrm{Nan} \mathrm{Li}^{6, \mathrm{f}}$ \\ ${ }^{1}$ School of Instrument Science and Opto-electronics Engineering, \\ HeFei University of Technology, P. R. China \\ ${ }^{2-6}$ College of Mechanical and Electronic Engineering, Nanjing Forestry University, P. R. China \\ ${ }^{a}$ cj1993@mail.hfut.edu.cn, bchenyongnj@sohu.com, chironking@gmail.com, \\ dichejun@163.com, egaofeng8960@126.com, ${ }^{\mathrm{f}} 943194662 @ q q . c o m$
} Keywords: Parallel Robot; Tea Flushes Plucking; Machine Vision; Fringe Projection Profilometry;
DSP

\begin{abstract}
Recent developed tea plucking machines mainly bases on the shearing principle which cut the top of the tea trees with no selectivity. Automatic tea flushes plucking machinery is essential to tea harvest for high-quality green tea manufacture, as labor cost increases rapidly in recent years. This paper focuses on the researches of a parallel green tea plucking robot with high efficiency. Color features are extracted to recognize tea flushes in the natural conditions using machine vision. Fringe projection profilometry is employed to realize $3 \mathrm{D}$ reconstruction and measurement for tea trees. A modified delta parallel robot with four arms is developed after kinematic analysis and mechanical design. The robot is controlled based on DSP (Digital Signal Processing). The experiments show over $85 \%$ could be detected successfully and the end-effector locating error is less than $2 \mathrm{~mm}$. The final objective of the researches is to realize a site-specific flushes plucking robot with high productivity which can replace several farms at green tea harvest season.
\end{abstract}

\section{Introduction}

In order to produce high-quality and high-grade green tea, only the tender flushes (consist of the bud and the first two leaves) are plucked in spring harvest season by hand. As the labor cost increases rapidly in recent years, labor cost accounts more and more in the total cost of tea manufacture[1]. Also, it is hard to find enough farmers to pluck tea flushes in time. Recent developed tea plucking machines mainly bases on the shearing principle which cut the top of the tea trees with no selectivity. In this way, the harvested leaves include old leaves and broken leaves, which only can be used to manufacture low grade tea. The plantation is eager for an automatic tea plucking machine which can only pluck the tender flushes to meet the demand of high-grade green tea manufacture.

Extensive researches on fruit and vegetable harvesting robots have been carried out all over the world in these years[2,3]. But there is less researches on the integent tea plucking machinary.Because of their high speed and high precision, Parallel robots have found their application in industry for many years. In this paper, a organial proposal is suggested to realize high-quality green tea plucking using a parallel robot and machine vision.

\section{Methods and Material}

According to the special demands on high-quality green tea plucking, we suggest a innvotive havest scheme as showed in Fig. 1. The robotics system[4] includs a CCD camera, a digital projector, a parallel robot, an end-effecor and an embedded controller. 
Color images are captured by the CCD camera over the tea trees. The color properties of the tea image were analyzed using luminance and color difference model. Grey level image was transformed into binary image using Otsu method and then area filter was employed to eliminate small noise regions. In this way, tea flushes were recognized and located ( $\mathrm{x}$ and y coordinates) in the natural conditions. In order to measure the height of the tea flush ( $\mathrm{z}$ coordinate), fringe projection profilometry was used to realize the 3D reconstruction for tea trees[5,6]. After the three coordinates were calcurated, they were sent to DSP controller and the robot was drived to pluck the tea flushes one by one just as a farmer does.

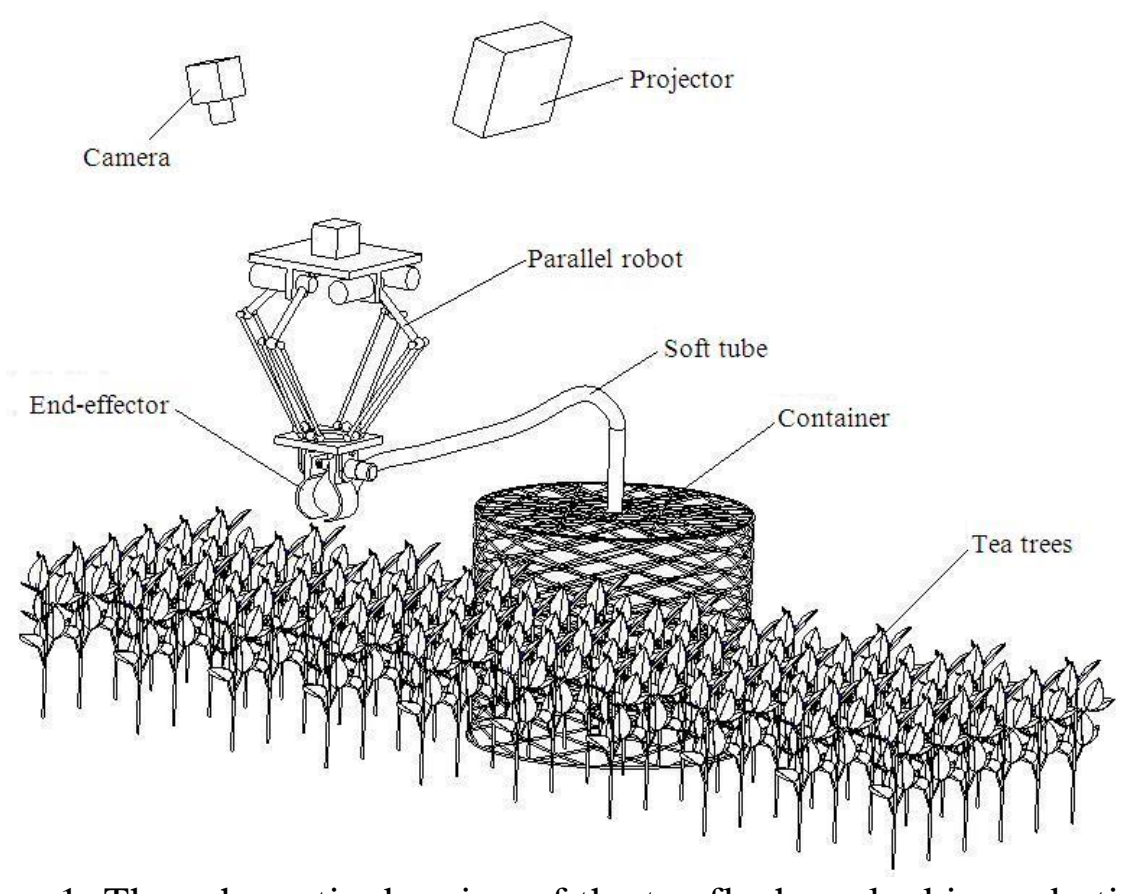

Figure. 1 The schematic drawing of the tea flushes plucking robotics system

In this research, sevesral color channeles and index were found to identificated tea flushes[7,8]. They were $y-c, y-m,(y-c) /(y+c)$, and $(y-m) /(y+m)$ in CMY; $S$ in HSI; U in YUV; B in LAB; Q in YIQ; i3 in i1i2i3; Yb in ArgYb; (R-B) in RGB; I in YIQ; $\mathrm{Cb}$ in $\mathrm{YcrCb}$; and so on. Fig. 2 shows one of the flowcharts for tea flushes identification.

The top plane of the tea trees is rough and discontinuous, so it is difficult to test the heights of the tea flushes respectively. In order to solve this problem, fringe projection profilometry was employed instead of ultrasonic and infrared techinques. A three-dimensional profilometry system based on fringe projection was built with a digital projector and a CCD camera. Measurement experiments of objects with discontinuous surface were carried out and the measurement system was optimized based on the localization requirements from tea flushes plucking robot. One of the outstanding features of some of the fringe projection techniques is their ability to provide high-resolution, whole-field 3D reconstruction of objects in a non-contact manner at video frame rates. This feature has backed the technique to pervade new areas of applications such as security systems, gaming and virtual reality[9]. Fig. 3 shows the flowchart for $3 \mathrm{D}$ reconstruction and measurement for tea trees.

The parallel mechanism and end effector were designed[10]. GUI optimization toolbox was used to determine the initial size of robot arms by the given workspace. The velocity Jacobi matrix and acceleration Jacobi matrix of the parallel robot were calculated through calculating the Jacobi matrix of high-quality tea parallel plucking robot by the vector method and derivation method. The mechanism singularity was analyzed according to the velocity Jacobi matrix and then the singularity positions of the robot were determined. The inverse kinematics was solved using the vector module and homogeneous coordinate transformation. The results verified the validity of the calculation of 
homogeneous coordinate transformation. Based on the inverse kinematics solution, the robot workspace were determined using the method that combining analytical method and numerical method. The 3D plot of the robot workspace was obtained through the plot command of MATLAB, and the robot workspace was proved to satisfy the design requirements. The model built by Pro/E software was imported into ADAMS simulation analytical software to analyze the robotic kinematics simulation. The kinematics inverse solution was accurately calculated by MATLAB, and the correctness of the simulation by ADAMS was testified. The forward kinematics simulation analysis of the robot was realized based on these resultes.

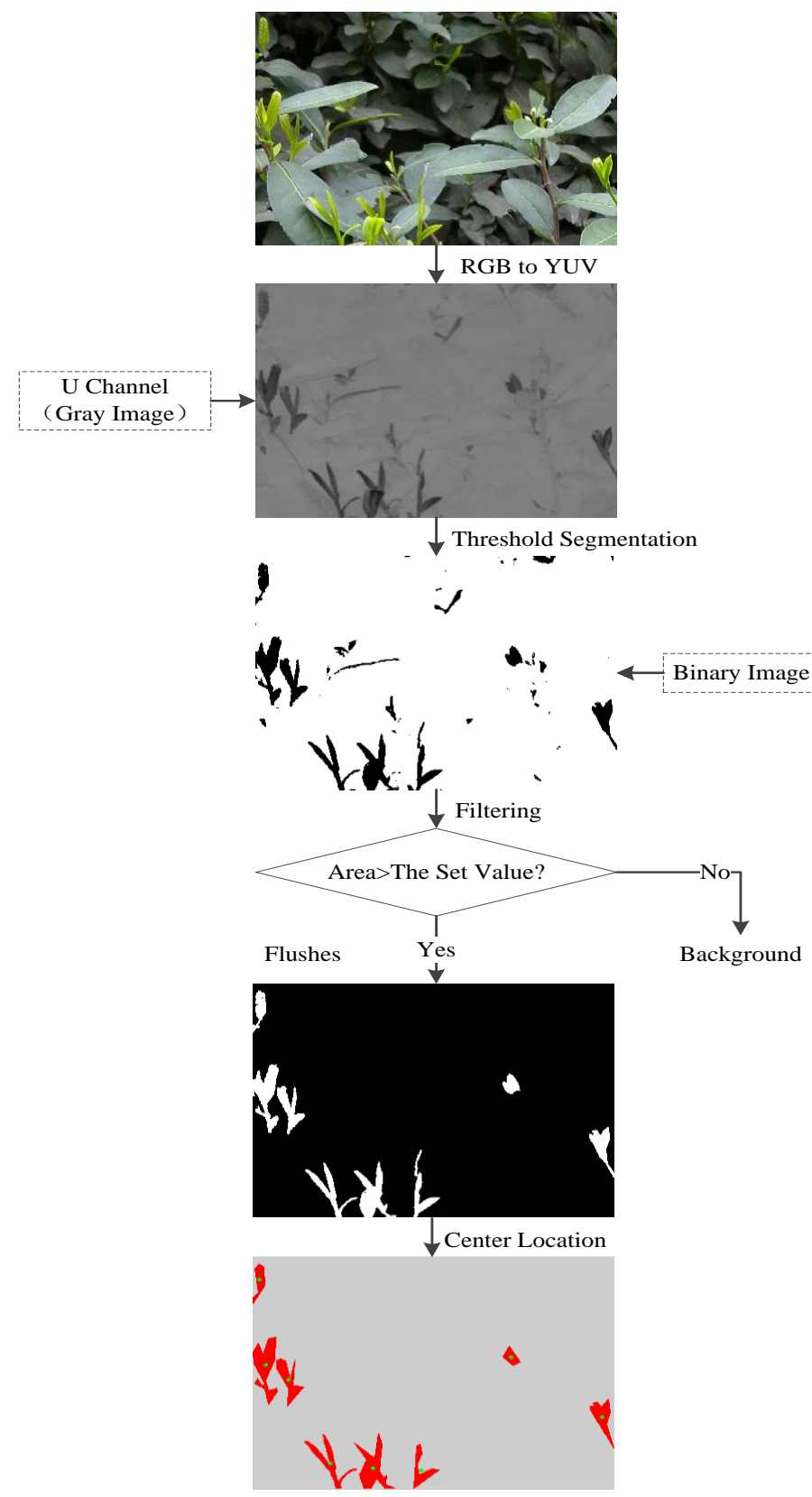

Figure. 2 The flowchart for green tea flushes identification

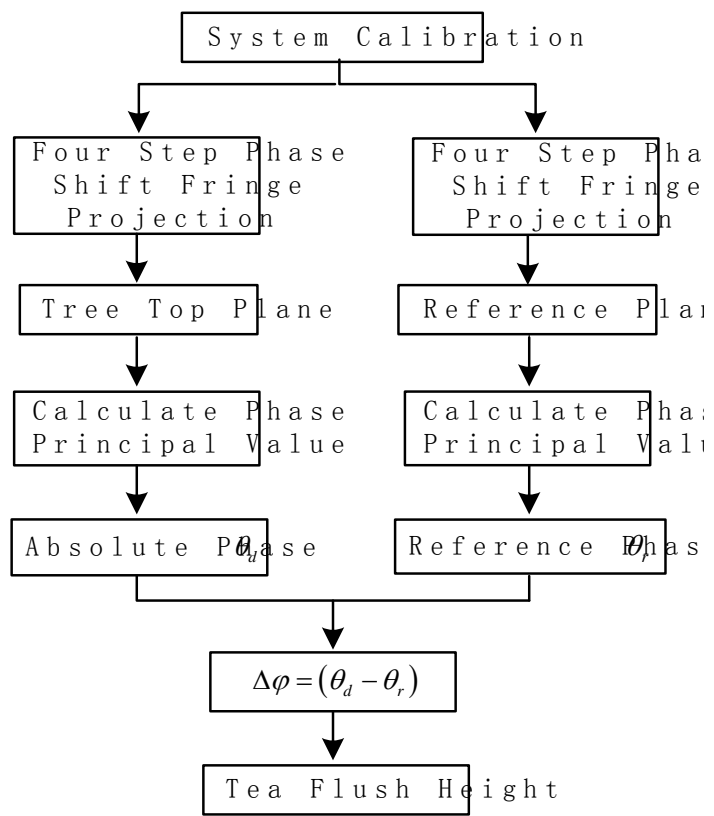

Figure. 3 The flowchart for $3 \mathrm{D}$ reconstruction and measurement for tea trees

\section{Results and discussion}

In this research, both hardware and software were developed and tested. The hardware includes a 
parallet robot and its control system. The software includes the tea flushes identification algorithms, $3 \mathrm{D}$ reconstruction algorithms for tea trees and so on. Fig. 4 shows the prototype of the parallel robot developed. Fig. 5 is the flowchart of the overall control system. Lab and field experiments show $85 \%$ spring green tea flushes could be detected successfully, and the height measurement error of tea flushes was less $3 \mathrm{~mm}$. The location error of the robot arm is less $2 \mathrm{~mm}$. These resultes can meet the demands for high-quality green tea plucking in the fields.
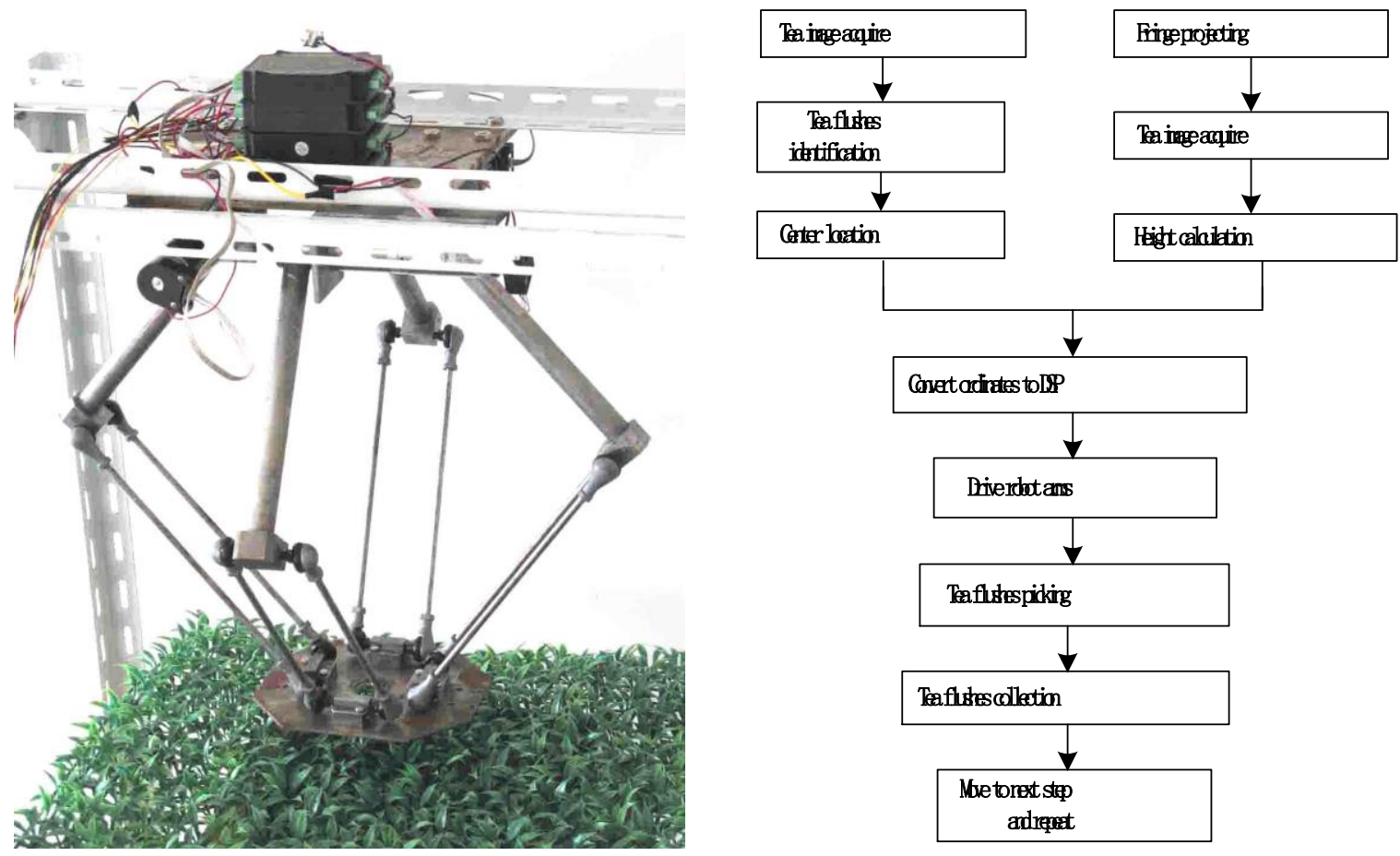

Figure. 4 The prototype of the parallel Figure. 5 The flowchart of the overall control robot robot

system

\section{Conclusion}

A prototype robotics system is developed based on active machine vision and DSP controller for high-quality green tea plucking in spring harvest season. Since the work is very complicated, there are several problems are still under reseaches. Tea farmers are looking forward to the intelligent green tea plucking machinery urgently.

\section{Acknowledgments}

This work was supported by National Key Technologies R \& D Program of China under grant number 2011BAD20B07.

\section{References}

[1] J.J. Wei, Y. Chen, X.J. Jin and et al: Journal of Tea Science, (2012) No.32, p.377.

[2] J. Song: Journal of Convergence Information Technology, (2011) No.6, p.41.

[3] D. Bulanon, T, Kataoka and Y. Ota: Biosystem Engineering, (2002) No.83, p.405.

[4] Y. Chen and J. Chen: Chinese Patent 2011103803978. (2011). (In Chinese) 
[5] H. Zhang, Y. Chen, W. Wang and et al: Transactions of the Chinese Society for Agricultural Machinery, (2014) No.45, p.61. (In Chinese)

[6] J. Che: Research and Application of Three-dimensional Profilometry Based on Fringe Projection, (MS., Nanjing Forestry University, China 2014), p.53. (In Chinese)

[7] X. J. Jin, Y. Chen, H. Zhang and et al: International Journal of Digital Content Technology and its Applications, (2012) No.6, p.600.

[8] X. J. Jin, Y. Chen, Y. Q. Guo and et al: Applied Mechanics and Materials, (2013) No.288, p.214.

[9] S. S. Gorthi, P. Rastogi: Optics and Lasers in Engineering, (2010) No.48, p.133.

[10]F. Gao: Structural Design and Simulation of a High-quality Tea Parallel Plucking Robot, (MS., Nanjing Forestry University, Chinese 2013), p.8. (In Chinese) 